Article

\title{
On the Lyapunov Exponent of Monotone Boolean Networks ${ }^{\dagger}$
}

\author{
Ilya Shmulevich \\ Institute for Systems Biology, Seattle, WA 98103, USA; ilya.shmulevich@isbscience.org \\ + Dedicated to the memory of A. D. Korshunov (1936-2019).
}

Received: 8 June 2020; Accepted: 23 June 2020; Published: date

check for updates

\begin{abstract}
Boolean networks are discrete dynamical systems comprised of coupled Boolean functions. An important parameter that characterizes such systems is the Lyapunov exponent, which measures the state stability of the system to small perturbations. We consider networks comprised of monotone Boolean functions and derive asymptotic formulas for the Lyapunov exponent of almost all monotone Boolean networks. The formulas are different depending on whether the number of variables of the constituent Boolean functions, or equivalently, the connectivity of the Boolean network, is even or odd.
\end{abstract}

Keywords: lyapunov exponent; monotone boolean function; boolean network

\section{Introduction}

Boolean networks are complex dynamical systems that were proposed as models of genetic regulatory networks $[1,2]$ and have since then been used to model a range of complex phenomena. Random Boolean networks (RBNs) are ensembles of randomly generated Boolean networks with random topology and random updating functions. It is known that RBNs undergo a phase transition. In the thermodynamic limit, meaning that the number of nodes goes to infinity, the phase transition curve for RBNs is given by $\lambda=\log (2 K p(1-p))=0$, where $p$ is the so-called bias of the random Boolean functions, which is the probability that the function takes on the value 1 , and $K$ is the connectivity of the network, or equivalently, the number of inputs to each Boolean function [3].

Under a synchronous updating scheme, whereby all Boolean functions get updated simultaneously at each time step, this phase transition curve separates two qualitatively distinct dynamical regimes. Below the curve, when $\lambda<0$, infinitesimally small perturbations to the state of the network decay, while above the curve, when $\lambda>0$, small perturbations spread throughout the network. Thus, the two regimes are often referred to as ordered and chaotic regimes, respectively. The quantity $\lambda$ coincides with the Lyapunov exponent $[4,5]$. Networks that are known to operate in the dynamically critical regime, meaning that their Lyapunov exponent is 0 , are widely known to have many optimal properties and are thought to be a hallmark of living systems [2,6]. In the context of Boolean networks, these properties include: the ability to balance resilience to random mutations with emergence of new phenotypes for adapting to new environmental challenges [7], maximization of the associative memory of the network [8], maximization of the diversity in structure-dynamics relationships [9], maximization of communication among nodes [10], maximization of so-called set complexity of the dynamics of the network [11], emergence of diversity in a spatial arrangement of Boolean networks representing cells in a tissue [12], and optimum network learning and generalization when the Boolean network has input and output signals [13,14].

We consider monotone Boolean networks in which all updating rules belong to the class of monotone Boolean functions. This class of functions is one of the most widely studied classes of Boolean functions [15]. Monotone Boolean networks have been primarily studied in the asynchronous 
updating scheme setting, whereby only one node is updated at a time. Some work has focused on long-term dynamics, such as fixed points and limit cycles [16-18]. It is also known that certain classes of fully asynchronous Boolean networks can be simulated by monotone Boolean networks [19]. However, in the context of the Lyapunov exponent, asynchronous updating schemes appear to be less relevant than synchronous updating schemes [20]. Our main results are asymptotic formulas, depending on whether $n$ is even or odd, for the so-called expected average sensitivity of a monotone Boolean function, first given in [21]. In light of the results in [5], the logarithm of the expected average sensitivities, $\hat{s}^{f}$, in Theorems 4 and 5 , can be directly interpreted as the Lyapunov exponent $\lambda$.

In the remainder, we will use $n$, rather than the conventional $K$ in the Boolean network literature, to denote the number of variables of the Boolean functions. Our results concerning almost all monotone Boolean networks can be understood in a probabilistic manner, meaning that the asymptotic formulas are valid with probability almost 1 if a monotone Boolean network is chosen at random from the set of all such networks. As the formulas are asymptotic, they should not be interpreted for small $n$. At the same time, they are quite accurate even for $n=7$, which follows from the results originally published in [22]. Absent additional constraints on the Boolean functions, such as the classes of canalizing functions [5,23-25] or Post classes [26], random monotone Boolean networks quickly enter the disordered regime relative to $n$, but slower than RBNs. For example, for $n=4$, monotone Boolean networks have the expected average sensitivity $\hat{s}^{f}=1.125$, which is already slightly in the chaotic regime $(\lambda>0)$. For $n=20$, the expected average sensitivity is $s^{f}=3.2$, whereas for random Boolean networks, it is $s^{f}=10$ (we can also see this if we plug $n=20$ and $p=1 / 2$ into $2 n p(1-p)$ ).

\section{Definitions and Preliminaries}

Let $f:\{0,1\}^{n} \rightarrow\{0,1\}$ be a Boolean function of $n$ variables $x_{1}, \ldots, x_{n}$. Let

$$
\partial f(\tilde{x}) / \partial x_{j}=f\left(\tilde{x}^{(j, 0)}\right) \oplus f\left(\tilde{x}^{(j, 1)}\right)
$$

be the partial derivative of $f$ with respect to $x_{j}$, where $\oplus$ is addition modulo 2 (exclusive OR) and $\tilde{x}^{(j, k)}=\left(x_{1}, \ldots, x_{j-1}, k, x_{j+1}, \ldots x_{n}\right), k=0,1$. Clearly, the partial derivative is a Boolean function itself that specifies whether a change in the $j$ th input causes a change in the original function $f$. The activity of variable $x_{j}$ in function $f$ can be defined as

$$
\alpha_{j}^{f}=\frac{1}{2^{n}} \sum_{\tilde{x} \in\{0,1\}^{n}} \partial f(\tilde{x}) / \partial x_{j}
$$

Note that although the vector $\tilde{x}$ consists of $n$ components (variables), the $j$ th variable is fictitious in $\partial f(\tilde{x}) / \partial x_{j}$. A variable $x_{j}$ is fictitious in $f$ if $f\left(\tilde{x}^{(j, 0)}\right)=f\left(\tilde{x}^{(j, 1)}\right)$ for all $\tilde{x}^{(j, 0)}$ and $\tilde{x}^{(j, 1)}$. For a $n$-variable Boolean function $f$, we can form its activity vector $\tilde{\alpha}^{f}=\left[\alpha_{1}^{f}, \ldots, \alpha_{n}^{f}\right]$. It is easy to see that $0 \leq \alpha_{j}^{f} \leq 1$, for any $j=1, \ldots, n$. In fact, we can consider $\alpha_{j}^{f}$ to be a probability that toggling the $j$ th input bit changes the function value, when the input vectors $\tilde{x}$ are distributed uniformly over $\{0,1\}^{n}$. Since we're in the binary setting, the activity is also the expectation of the partial derivative with respect to the uniform distribution: $\alpha_{j}^{f}=E\left[\partial f(\tilde{x}) / \partial x_{j}\right]$. The activity of a fictitious variable $x_{j}$ is $\alpha_{j}^{f}=0$. Under an arbitrary distribution, $\alpha_{j}^{f}$ is referred to as the influence of variable $x_{j}$ on the function $f$ [27]. The influence of variables was used in the context of genetic regulatory network modeling in [28].

Another important quantity is the sensitivity of a Boolean function $f$, which measures how sensitive the output of the function is to changes in the inputs. The sensitivity $s^{f}(\tilde{x})$ of $f$ on vector $\tilde{x}$ is 
defined as the number of Hamming neighbors of $\tilde{x}$ on which the function value is different than on $\tilde{x}$ (two vectors are Hamming neighbors if they differ in only one component). That is,

$$
\begin{aligned}
s^{f}(\tilde{x}) & =\left|\left\{i \in\{1, \ldots, n\}: f\left(\tilde{x} \oplus e_{i}\right) \neq f(\tilde{x})\right\}\right| \\
& =\sum_{i=1}^{n} \chi\left[f\left(\tilde{x} \oplus e_{i}\right) \neq f(\tilde{x})\right],
\end{aligned}
$$

where $e_{i}$ is the unit vector with 1 in the $i$ th position and 0 s everywhere else and $\chi[A]$ is an indicator function that is equal to 1 if and only if $A$ is true. The average sensitivity $s^{f}$ is defined by taking the expectation of $s^{f}(\tilde{x})$ with respect to the distribution of $\tilde{x}$. It is easy to see that under the uniform distribution, the average sensitivity is equal to the sum of the activities:

$$
\begin{aligned}
s^{f} & =E\left[s^{f}(\tilde{x})\right]=\sum_{i=1}^{n} E\left[\chi\left[f\left(\tilde{x} \oplus e_{i}\right) \neq f(\tilde{x})\right]\right] \\
& =\sum_{i=1}^{n} \alpha_{i}^{f} .
\end{aligned}
$$

Therefore, $s^{f}$ is a number between 0 and $n$.

The average sensitivity has been studied intensively by a number of authors [5,29-37]. For example, it was shown by Friedgut [33] that if the average sensitivity of $f$ is $k$ then $f$ can be approximated by a function depending on only $c^{k}$ variables where $c$ is a constant depending only on the accuracy of the approximation, but not on $n$. Shi [34] showed that the average sensitivity can serve as a lower bound of quantum query complexity. Average sensitivity was used to characterize the noise sensitivity of monotone Boolean functions by Mossel and O'Donnell [35]. Zhang [36] gives lower and upper bounds of the average sensitivity of a monotone Boolean function. The upper bound is asymptotic to $\sqrt{n}$, which has been shown by Bshouty and Tamon [37]. Shmulevich and Kauffman [5] have shown that the average sensitivity determines the critical phase transition curve in random Boolean networks and thus coincides with the Lyapunov exponent as $\lambda=\log s^{f}$. We now turn to monotone Boolean functions.

\subsection{Monotone Boolean Functions}

Let $\tilde{\alpha}=\left(\alpha_{1}, \cdots, \alpha_{n}\right)$ and $\tilde{\beta}=\left(\beta_{1}, \cdots, \beta_{n}\right)$ be two different $n$-element binary vectors. We say that $\tilde{\alpha}$ precedes $\tilde{\beta}$, denoted as $\tilde{\alpha} \prec \tilde{\beta}$, if $\alpha_{i} \leq \beta_{i}$ for every $i, 1 \leq i \leq n$. If $\tilde{\alpha} \nprec \tilde{\beta}$ and $\tilde{\beta} \nprec \tilde{\alpha}$, then $\tilde{\alpha}$ and $\tilde{\beta}$ are said to be incomparable. Relative to the predicate $\prec$, the set of all binary vectors of a given length is a partially ordered set. A Boolean function $f\left(x_{1}, \cdots, x_{n}\right)$ is called monotone if for any two vectors $\tilde{\alpha}$ and $\tilde{\beta}$ such that $\tilde{\alpha} \prec \tilde{\beta}$, we have $f(\tilde{\alpha}) \leq f(\tilde{\beta})$.

We denote by $M(n)$ the set of all monotone Boolean functions of $n$ variables. Let $E^{n}$ denote the Boolean $n$-cube, that is, a graph with $2^{n}$ vertices each of which is labeled by an $n$-element binary vector. Two vertices $\tilde{\alpha}=\left(\alpha_{1}, \cdots, \alpha_{n}\right)$ and $\tilde{\beta}=\left(\beta_{1}, \cdots, \beta_{n}\right)$ are connected by an edge if and only if the Hamming distance $\rho(\tilde{\alpha}, \tilde{\beta})=\sum_{i=1}^{n}\left(\alpha_{i} \oplus \beta_{i}\right)=1$. The set of those vectors from $E^{n}$ in which there are exactly $k$ units, $0 \leq k \leq n$, is called the $k$ th layer of $E^{n}$ and is denoted by $E^{n, k}$.

A vector $\tilde{\alpha} \in E^{n}$ is called a minimal one or minimal unit of monotone Boolean function $f\left(x_{1}, \ldots, x_{n}\right)$ if $f(\tilde{\alpha})=1$ and $f(\tilde{\beta})=0$ for any $\tilde{\beta} \prec \tilde{\alpha}$. A vector $\tilde{\alpha} \in E^{n}$ is called an maximal zero of monotone Boolean function $f\left(x_{1}, \ldots, x_{n}\right)$ if $f(\tilde{\alpha})=0$ and $f(\tilde{\beta})=1$ for any $\tilde{\beta} \succ \tilde{\alpha}$. The minimal ones correspond directly to the terms in the minimal disjunctive normal form (DNF) representation of the monotone Boolean function. In [38], asymptotic formulae for the number of monotone Boolean functions of $n$ variables with a most probable number of minimal ones were derived, confirming the conjecture in [39] that the number of monotone Boolean functions relative to the number of minimal ones asymptotically follows a normal distribution, with the assumption of all monotone Boolean functions being equiprobable. 


\subsection{The Structure of Special Monotone Boolean Functions}

We now briefly review some known results concerning the structure of so-called special monotone Boolean functions. Let $M_{0}(n)$ denote the set of functions in $M(n)$ possessing the following properties. If $n$ is even, then $M_{0}(n)$ contains only functions $f \in M(n)$ such that all minimal ones of $f$ are situated in $E^{n, n / 2-1}, E^{n, n / 2}$, and $E^{n, n / 2+1}$ while function $f$ is equal to 1 on all vectors in $E^{n, n / 2+2}, \cdots, E^{n, n}$. For odd $n, M_{0}(n)$ contains only functions $f \in M(n)$ such that all minimal ones of $f$ are situated in either $E^{n,(n-3) / 2}, E^{n,(n-1) / 2}$, and $E^{n,(n+1) / 2}$ or $E^{n,(n-1) / 2}, E^{n,(n+1) / 2}$, and $E^{n,(n+3) / 2}$. In the first case, $f(\tilde{\alpha})=1$ for all $\tilde{\alpha}$ in $E^{n,(n+3) / 2}, \ldots, E^{n, n}$ while in the second case, $f(\tilde{\alpha})=1$ for all $\tilde{\alpha}$ in $E^{n,(n+5) / 2}, \ldots, E^{n, n}$.

Then, as shown in [22],

$$
\lim _{n \rightarrow \infty} \frac{\left|M_{0}(n)\right|}{|M(n)|}=1
$$

which we denote by $\left|M_{0}(n)\right| \sim|M(n)|$. In [40], asymptotic formulae for the number of special functions from $M_{0}(n)$ were established and subsequently used to characterize statistical properties of a popular class of nonlinear digital filters called stack filters [41]. The set of these special functions is denoted by $M_{0}^{1}(n)$ and, depending on whether $n$ is even or odd, is defined differently. While we shall omit the rather lengthy definitions of special functions, the result from [40] that will be important to us is that $\left|M_{0}^{1}(n)\right| \sim|M(n)|$. In other words, almost all monotone Boolean functions are special. We shall also need the following results.

Let us start with the case of even $n$. Let

$$
\begin{aligned}
& r_{0}=r_{0}(n)=v_{0}=v_{0}(n)=\left\lfloor\left(\begin{array}{c}
n \\
n / 2-1
\end{array}\right) 2^{-n / 2-1}\right\rfloor, \\
& z_{0}=\left\lfloor\frac{1}{2}\left(\begin{array}{c}
n \\
n / 2
\end{array}\right)\right\rfloor .
\end{aligned}
$$

Let $M_{0}^{1}(n, r, z, v)$ denote the set of functions $f \in M_{0}^{1}(n)$ such that $f$ has $r$ minimal ones in $E^{n, n / 2-1}$, $v$ maximal zeros in $E^{n, n / 2+1}$, and $f$ is equal to 1 on $z$ vertices in $E^{n, n / 2}$. In [40], the following result was proved.

Theorem 1. Let $n$ be even,

$$
r=r_{0}+k, z=z_{0}+u, v=v_{0}+t,
$$

where $r_{0}, z_{0}, v_{0}$ are defined in (6). Then, for any $k, t$, and $u$ such that $|k| \leq n 2^{n / 4},|t| \leq n 2^{n / 4},|u| \leq n 2^{n / 2}$,

$$
\begin{aligned}
\left|M_{0}^{1}(n, r, z, v)\right| \sim \sqrt{\frac{2^{n+1}}{\pi^{3}\left(\begin{array}{c}
n \\
n / 2
\end{array}\right)^{3}}}|M(n)| & \quad \times \exp \left\{-\frac{2^{n / 2}}{\left(\begin{array}{c}
n \\
n / 2-1
\end{array}\right)}\left(k^{2}+t^{2}\right)-\frac{2 u^{2}}{\left(\begin{array}{c}
n \\
n / 2
\end{array}\right)}\right\} .
\end{aligned}
$$

For any odd $n$, we use the parameters $r_{1}, z_{1}, v_{1}$ which are given by

$$
\begin{aligned}
& r_{1}=r_{1}(n)=\left\lfloor\left(\begin{array}{c}
n \\
(n-3) / 2
\end{array}\right) 2^{-(n+3) / 2}\right\rfloor, \\
& v_{1}=v_{1}(n)=\left\lfloor\left(\begin{array}{c}
n \\
(n+1) / 2
\end{array}\right) 2^{-(n+1) / 2}\right\rfloor, \\
& z_{1}=\left\lfloor\frac{1}{2}\left(\left(\begin{array}{c}
n \\
(n-1) / 2
\end{array}\right)+r_{1}(n+3) / 2-v_{1}(n+1) / 2\right)\right\rfloor
\end{aligned}
$$


and parameters $r_{2}, z_{2}, v_{2}$, which are given by

$$
\begin{aligned}
& r_{2}=r_{2}(n)=\left\lfloor\left(\begin{array}{c}
n \\
(n-1) / 2
\end{array}\right) 2^{-(n+1) / 2}\right\rfloor, \\
& v_{2}=v_{2}(n)=\left\lfloor\left(\begin{array}{c}
n \\
(n+3) / 2
\end{array}\right) 2^{-(n+3) / 2}\right\rfloor, \\
& z_{2}=\left\lfloor\frac{1}{2}\left(\left(\begin{array}{c}
n \\
(n+1) / 2
\end{array}\right)+r_{2}(n-1) / 2-v_{2}(n+3) / 2\right)\right\rfloor .
\end{aligned}
$$

Let $M_{0,1}^{1}(n, r, z, v)$ denote the set of functions $f \in M_{0}^{1}(n)$ such that $f$ has $r$ minimal ones in $E^{n,(n-3) / 2}, v$ maximal zeros in $E^{n,(n+1) / 2}$, and $f$ is equal to 1 on $z$ vertices in $E^{n,(n-1) / 2}$. Similarly, let $M_{0,2}^{1}(n, r, z, v)$ denote the set of functions $f \in M_{0}^{1}(n)$ such that $f$ has $r$ minimal ones in $E^{n,(n-1) / 2}$, $v$ maximal zeros in $E^{n,(n+3) / 2}$, and $f$ is equal to 1 on $z$ vertices in $E^{n,(n+1) / 2}$. Then, in [40], the following two Theorems were proved.

Theorem 2. Let $n$ be odd,

$$
r=r_{1}+k, z=z_{1}+u, v=v_{1}+t,
$$

where $r_{1}, z_{1}, v_{1}$ are defined in (8) and (9). Then, for any $k, t$, and $u$ such that $|k| \leq n 2^{n / 4},|t| \leq n 2^{n / 4}$, $|u| \leq n 2^{n / 2}$,

$$
\left|M_{0,1}^{1}(n, r, z, v)\right| \sim \frac{1}{2} \sqrt{\frac{2^{n+1}}{\pi^{3}\left(\begin{array}{c}
n \\
(n-1) / 2
\end{array}\right)^{3}}}|M(n)|
$$

$$
\times \exp \left\{-\frac{2^{(n+1) / 2}}{\left(\begin{array}{c}
n \\
(n-3) / 2
\end{array}\right)} k^{2}-\frac{2^{(n-1) / 2}}{\left(\begin{array}{c}
n \\
(n+1) / 2
\end{array}\right)} t^{2}-\frac{2 u^{2}}{\left(\begin{array}{c}
n \\
(n-1) / 2
\end{array}\right)}\right\} .
$$

Theorem 3. Let $n$ be odd,

$$
r=r_{2}+k, z=z_{2}+u, v=v_{2}+t
$$

where $r_{2}, z_{2}, v_{2}$ are defined in (10) and (11). Then, for any $k, t$, and $u$ such that $|k| \leq n 2^{n / 4},|t| \leq n 2^{n / 4}$, $|u| \leq n 2^{n / 2}$,

$$
\begin{aligned}
\left|M_{0,2}^{1}(n, r, z, v)\right| \sim \frac{1}{2} \sqrt{\frac{2^{n+1}}{\pi^{3}\left(\begin{array}{c}
n \\
(n-1) / 2
\end{array}\right)^{3}}}|M(n)| & \\
& \times \exp \left\{-\frac{2^{(n-1) / 2}}{\left(\begin{array}{c}
n \\
(n-1) / 2
\end{array}\right)} k^{2}-\frac{2^{(n+1) / 2}}{\left(\begin{array}{c}
n \\
(n+3) / 2
\end{array}\right)} t^{2}-\frac{2 u^{2}}{\left(\begin{array}{c}
n \\
(n+1) / 2
\end{array}\right)}\right\} .
\end{aligned}
$$

Prior to presenting the main results, we summarize some of the notation in Table 1.

Table 1. Table of notation.

\begin{tabular}{ll}
\hline$f$ & Boolean function \\
$n$ & number of variables of $f$ \\
$\partial f(\tilde{x}) / \partial x_{j}$ & partial derivative of $f$ with respect to $x_{j}$ \\
$\tilde{x}=\left(x_{1}, \ldots, x_{n}\right)$ & vector of $n$ binary values \\
$\alpha_{j}^{f}$ & activity of variable $x_{j}$ \\
$s^{f}$ & average sensitivity of a Boolean function $f$ \\
$\rho(\tilde{\alpha}, \tilde{\beta})$ & Hamming distance between $\tilde{\alpha}$ and $\tilde{\beta}$ \\
$M(n)$ & set of monotone Boolean functions of $n$ variables \\
$M_{0}(n)$ & set of special monotone Boolean functions of $n$ variables \\
$E^{n}$ & Boolean $n$-cube or $\{0,1\}^{n}$ with vertices $\tilde{x}$ \\
$E^{n, k}$ & $\left\{\tilde{x} \in E^{n} \mid \rho(\tilde{x}, \tilde{0})=k\right\}$ or the $k$ th layer of $E^{n}$ \\
\hline
\end{tabular}




\section{Main Results}

Since $\left|M_{0}(n)\right| \sim|M(n)|$, we can focus our attention on functions in $M_{0}(n)$ and derive the average sensitivity of a typical function from $M_{0}(n)$. By 'typical' we mean the most probable Boolean function relative to the parameters $k, t$, and $u$ in Theorems $1-3$. It can easily be seen that the most probable special Boolean functions will have $k=t=u=0$. This will imply, to take the $n$-even case as an example, that the most probable function $f$ has $r_{0}$ minimal ones in $E^{n, n / 2-1}, v_{0}$ maximal zeros in $E^{n, n / 2+1}$, and $f$ is equal to 1 on $z_{0}$ vertices in $E^{n, n / 2}$, where $r_{0}, v_{0}$, and $z_{0}$ are given in (6). Our proofs are thus based on the derivation of the average sensitivity of such a function. Whenever we make probabilistic assertions using words such as 'most probable' or 'typical' or talk about expectations, we are implicitly endowing the set $M_{0}^{1}(n, r, z, v)$ with a uniform probability distribution for fixed parameters $n, r, z, v$. This should not be confused with the Gaussian-like distribution of $M_{0}^{1}(n, r, z, v)$ relative to its parameters $n, r, z, v$ [38]. We will also omit the floor notation $\lfloor\cdot\rfloor$ as the results are asymptotic.

Theorem 4. Let $n$ be even and let $f \in M_{0}^{1}(n)$ be a typical monotone Boolean function. Then, the expected average sensitivity $\hat{s}^{f}=E\left[s^{f}\right]$ of $f$ is

$$
\hat{s}^{f} \sim n 2^{-n}\left(\begin{array}{c}
n \\
n / 2-1
\end{array}\right)\left(2^{-n / 2-1}+1\right) .
$$

Proof. We will proceed by first focusing on determining the activity of an arbitrary variable $x_{j}$ of a typical function $f$. By simple symmetry arguments, if we were to sample randomly from the set $M(n)$ of monotone Boolean functions, the expected activities would be equal for all the variables. It will follow by (4) that the expected average sensitivity will be equal to $n$ multiplied by the expected activity. Since the function $f$ is such that its minimal ones are situated in $E^{n, n / 2-1}, E^{n, n / 2}$, and $E^{n, n / 2+1}$ while it is equal to 1 on all vectors in $E^{n, n / 2+2}, \ldots, E^{n, n}$, the only non-trivial behavior occurs between the layers $E^{n, n / 2-2}$ and $E^{n, n / 2+2}$.

Let us consider the minimal ones, and hence all of the ones, on $E^{n, n / 2-1}$. Since we are considering variable $x_{j}$, half of these minimal ones will have $x_{j}=0$ (i.e., $\left.\tilde{x}^{(j, 0)}\right)$ and the other half will have $x_{j}=1$ (i.e., $\left.\tilde{x}^{(j, 1)}\right)$. It is easy to see that if $\tilde{x}^{(j, 0)} \in E^{n, n / 2-1}$ is a minimal one, then by monotonicity, $f\left(\tilde{x}^{(j, 1)}\right)=1$. Consequently, the Hamming neighbors $\tilde{x}^{(j, 0)}$ and $\tilde{x}^{(j, 1)}$ contribute nothing to the sum in (2). On the other hand, if $\tilde{x}^{(j, 1)} \in E^{n, n / 2-1}$ is a minimal one, then $\partial f(\tilde{x}) / \partial x_{j}=1$, since $f(\tilde{x})=0$ for all $\tilde{x} \in E^{n, n / 2-2}$. The most probable number of such minimal ones on $E^{n, n / 2-1}$ contributing to the sum in (2) is thus equal to

$$
\frac{1}{2}\left(\begin{array}{c}
n \\
n / 2-1
\end{array}\right) 2^{-n / 2-1}
$$

The number of zeros on $E^{n, n / 2-1}$ is equal to

$$
\left(\begin{array}{c}
n \\
n / 2-1
\end{array}\right)-r_{0}=\left(\begin{array}{c}
n \\
n / 2-1
\end{array}\right)\left(1-2^{-n / 2-1}\right)
$$

As above, half of these will have $x_{j}=0$ and half will have $x_{j}=1$. We need not consider vectors $\tilde{x}^{(j, 1)} \in E^{n, n / 2-1}$, since $f(\tilde{x})=0$ for all $\tilde{x} \in E^{n, n / 2-2}$. However, we should consider the number of ones situated on the middle layer $E^{n, n / 2}$. The middle layer contains

$$
\frac{1}{2}\left(\begin{array}{c}
n \\
n / 2
\end{array}\right)
$$


ones and an equal number of zeros. Thus, half of the vectors $\tilde{x}^{(j, 1)} \in E^{n, n / 2}$ will be ones and the other half will be zeros. In total, the number of vectors $\tilde{x} \in E^{n, n / 2-1}$ such that $f(\tilde{x})=0, x_{j}=0$, and $f\left(\tilde{x}^{(j, 1)}\right)=1$, is equal to

$$
\frac{1}{4}\left(\begin{array}{c}
n \\
n / 2-1
\end{array}\right)\left(1-2^{-n / 2-1}\right)
$$

We have now examined all partial derivatives above and below the layer $E^{n, n / 2-1}$.

Let us now jump to layer $E^{n, n / 2+1}$, as it will be similar by duality considerations. The number of maximal zeros on that layer is equal to $v_{0}=r_{0}$. Half of these will have $x_{j}=1$ and thus $\partial f(\tilde{x}) / \partial x_{j}=0$ due to monotonicity. The other half will have $x_{j}=0$ and since $f(\tilde{x})=1$ for all $\tilde{x} \in E^{n, n / 2+2}$, the same total as in (14) will result. Similarly, the number of ones on $E^{n, n / 2+1}$ is the same as in (15). We are only concerned with $\tilde{x}^{(j, 1)} \in E^{n, n / 2+1}$, such that $f\left(\tilde{x}^{(j, 1)}\right)=1$ and $f\left(\tilde{x}^{(j, 0)}\right)=0$. As above, because the middle layer contains the same number of ones and zeros, the total number of such pairs of vectors is the same as in (17). Thus, having accounted for all partial derivatives above and below $E^{n, n / 2+1}$ and having convinced ourselves that their contribution to the overall activity of variable $x_{j}$ is the same as in (14) and (17), we can multiply (14) and (17) by 2 and add them together to obtain

$$
\begin{aligned}
& \left(\begin{array}{c}
n \\
n / 2-1
\end{array}\right) 2^{-n / 2-1}+\frac{1}{2}\left(\begin{array}{c}
n \\
n / 2-1
\end{array}\right)\left(1-2^{-n / 2-1}\right) \\
= & \frac{1}{2}\left(\begin{array}{c}
n \\
n / 2-1
\end{array}\right)\left(2^{-n / 2-1}+1\right)
\end{aligned}
$$

Since there are $2^{-n+1}$ Hamming neighbors $\tilde{x}^{(j, 0)} \prec \tilde{x}^{(j, 1)}$ and since the average sensitivity is $n$ times the activity, we must multiply (19) by $n 2^{-n+1}$, resulting in the statement of the theorem.

The case of odd $n$ is somewhat more involved because there is no "middle" layer $E^{n, n / 2}$. Instead, typical functions break up into two sets: $M_{0,1}^{1}(n, r, z, v)$ and $M_{0,2}^{1}(n, r, z, v)$. In the first case, all minimal ones are on layers $E^{n,(n-3) / 2}, E^{n,(n-1) / 2}$, and $E^{n,(n+1) / 2}$ while in the second case, all minimal ones are situated on $E^{n,(n-1) / 2}, E^{n,(n+1) / 2}$, and $E^{n,(n+3) / 2}$. Under random sampling, these two cases will occur with equal probabilities. As we shall see, the results will be different for each case. Thus, the expected average sensitivity will be the average of the the expected average sensitivities corresponding to these two cases.

Theorem 5. Let $n$ be odd and let $f \in M_{0}^{1}(n)$ be a typical monotone Boolean function. Then, the expected average sensitivity $\hat{s}^{f}=E\left[s^{f}\right]$ of $f$ is

$$
\hat{s}^{f} \sim \frac{1}{2}\left(\hat{s}_{1}^{f}+\hat{s}_{2}^{f}\right),
$$

where $\hat{s}_{1}^{f}$ and $\hat{s}_{2}^{f}$ are given in (28) and (37), respectively.

Proof. Let us first address the set $M_{0,1}^{1}(n, r, z, v)$. As in Theorem 4, we only need to concern ourselves with four cases. These are:

1. $\tilde{x} \in E^{n,(n-3) / 2}, f(\tilde{x})=1, x_{j}=1$

2. $\tilde{x} \in E^{n,(n-3) / 2}, f(\tilde{x})=0, x_{j}=0$

3. $\tilde{x} \in E^{n,(n+1) / 2}, f(\tilde{x})=1, x_{j}=1$

4. $\tilde{x} \in E^{n,(n+1) / 2}, f(\tilde{x})=0, x_{j}=0$

All other situations, such as $\tilde{x} \in E^{n,(n-3) / 2}, f(\tilde{x})=1, x_{j}=0$, will result in the partial derivatives being zero due to monotonicity of $f$, hence will make no contribution to the activity of variable $x_{j}$. There are

$$
\frac{1}{2}\left(\begin{array}{c}
n \\
(n-3) / 2
\end{array}\right) 2^{-(n+3) / 2}
$$


minimal ones on $E^{n,(n-3) / 2}$ such that $x_{j}=1$. At the same time, there are

$$
\frac{1}{2}\left(\begin{array}{c}
n \\
(n-3) / 2
\end{array}\right)\left(1-2^{-(n+3) / 2}\right)
$$

zeros on $E^{n,(n-3) / 2}$ such that $x_{j}=0$. Unlike in the $n$-even case, where the middle layer contains an equal number of ones and zeros, the number of ones and zeros on $E^{n,(n-1) / 2}$ is not equal. The number of ones on $E^{n,(n-1) / 2}$ is given in (9). Thus, if $\tilde{x}^{(j, 0)}$ is a zero on $E^{n,(n-3) / 2}$, then the probability that $f\left(\tilde{x}^{(j, 1)}\right)=1$ is

$$
\begin{aligned}
\left(\begin{array}{c}
n \\
(n-1) / 2
\end{array}\right)^{-1}\left(\frac { 1 } { 2 } \left(\left(\begin{array}{c}
n \\
(n-1) / 2
\end{array}\right)+\left(\begin{array}{c}
n \\
(n-3) / 2
\end{array}\right)\right.\right. & 2^{-(n+3) / 2}(n+3) / 2 \\
& \left.\left.-\left(\begin{array}{c}
n \\
(n+1) / 2
\end{array}\right) 2^{-(n+1) / 2}(n+1) / 2\right)\right)
\end{aligned}
$$

where we have simply divided the number of ones on $E^{n,(n-1) / 2}$ by the total number of vectors on that layer. Thus, multiplying (21) by (22) and adding to (20) gives us the total contribution to the activity of variable $x_{j}$ from cases 1 and 2 above, which is equal to

$$
\begin{array}{r}
\frac{1}{2}\left(\begin{array}{c}
n \\
(n-3) / 2
\end{array}\right) 2^{-(n+3) / 2}+\frac{1}{2}\left(\begin{array}{c}
n \\
(n-3) / 2
\end{array}\right)\left(1-2^{-(n+3) / 2}\right) \times \\
\left.\left.\left(\begin{array}{c}
n \\
(n-1) / 2
\end{array}\right)^{-1}\left(\begin{array}{r}
\frac{1}{2}\left(\left(\begin{array}{c}
n \\
(n-1) / 2
\end{array}\right)+\right. \\
\quad-\left(\begin{array}{c}
n \\
(n-3) / 2
\end{array}\right) 2^{-(n+3) / 2}(n+3) / 2 \\
(n+1) / 2
\end{array}\right) 2^{-(n+1) / 2}(n+1) / 2\right)\right)
\end{array}
$$

Cases 3 and 4 are similar. The number of (maximal) zeros on $E^{n,(n+1) / 2}$ for which $x_{j}=0$ is equal to

$$
\frac{1}{2}\left(\begin{array}{c}
n \\
(n+1) / 2
\end{array}\right) 2^{-(n+1) / 2}
$$

and the number of ones on $E^{n,(n+1) / 2}$ for which $x_{j}=1$ is equal to

$$
\frac{1}{2}\left(\begin{array}{c}
n \\
(n+1) / 2
\end{array}\right)\left(1-2^{-(n+1) / 2}\right)
$$

The proportion of zeros on $E^{n,(n-1) / 2}$ is

$$
\begin{aligned}
&\left(1-\left(\begin{array}{c}
n \\
(n-1) / 2
\end{array}\right)^{-1}\left(\frac { 1 } { 2 } \left(\left(\begin{array}{c}
n \\
(n-1) / 2
\end{array}\right)+\right.\right.\right. \\
&\left.\left.\left.\left(\begin{array}{c}
n \\
(n-3) / 2
\end{array}\right) 2^{-(n+3) / 2}(n+3) / 2-\left(\begin{array}{c}
n \\
(n+1) / 2
\end{array}\right) 2^{-(n+1) / 2}(n+1) / 2\right)\right)\right)
\end{aligned}
$$


Thus, as before, multiplying (25) by (26) and adding to (24), we get the total contribution to the activity from above and below the layer $E^{n,(n+1) / 2}$ (cases 3 and 4), resulting in

$$
\begin{aligned}
& \frac{1}{2}\left(\begin{array}{c}
n \\
(n+1) / 2
\end{array}\right) 2^{-(n+1) / 2}+ \frac{1}{2}\left(\begin{array}{c}
n \\
(n+1) / 2
\end{array}\right)\left(1-2^{-(n+1) / 2}\right) \times \\
&\left(1-\left(\begin{array}{c}
n \\
(n-1) / 2
\end{array}\right)\right.\left(\frac { 1 } { 2 } \left(\left(\begin{array}{c}
n \\
(n-1) / 2
\end{array}\right)+\right.\right. \\
&\left.\left.\left.\left(\begin{array}{c}
n \\
(n-3) / 2
\end{array}\right) 2^{-(n+3) / 2}(n+3) / 2-\left(\begin{array}{c}
n \\
(n+1) / 2
\end{array}\right) 2^{-(n+1) / 2}(n+1) / 2\right)\right)\right)
\end{aligned}
$$

Finally, adding (23) and (27) and then multiplying by $n 2^{-n+1}$ as in Theorem 4, we obtain the expected average sensitivity $\hat{s}_{1}^{f}$ of a typical function from $M_{0,1}^{1}(n, r, z, v)$ :

$$
\begin{aligned}
& \hat{s}_{1}^{f} \sim n 2^{-n+1}\left(\frac{1}{2}\left(\begin{array}{c}
n \\
(n-3) / 2
\end{array}\right) 2^{-(n+3) / 2}+\frac{1}{2}\left(\begin{array}{c}
n \\
(n-3) / 2
\end{array}\right)\left(1-2^{-(n+3) / 2}\right) \times\right. \\
& \left(\begin{array}{c}
n \\
(n-1) / 2
\end{array}\right)^{-1}\left(\frac { 1 } { 2 } \left(\left(\begin{array}{c}
n \\
(n-1) / 2
\end{array}\right)+\left(\begin{array}{c}
n \\
(n-3) / 2
\end{array}\right) 2^{-(n+3) / 2}(n+3) / 2\right.\right. \\
& \left.\left.-\left(\begin{array}{c}
n \\
(n+1) / 2
\end{array}\right) 2^{-(n+1) / 2}(n+1) / 2\right)\right)+ \\
& \frac{1}{2}\left(\begin{array}{c}
n \\
(n+1) / 2
\end{array}\right) 2^{-(n+1) / 2}+\frac{1}{2}\left(\begin{array}{c}
n \\
(n+1) / 2
\end{array}\right)\left(1-2^{-(n+1) / 2}\right) \times \\
& \left(1-\left(\begin{array}{c}
n \\
(n-1) / 2
\end{array}\right)^{-1}\left(\frac { 1 } { 2 } \left(\left(\begin{array}{c}
n \\
(n-1) / 2
\end{array}\right)+\right.\right.\right. \\
& \left.\left.\left.\left.\left(\begin{array}{c}
n \\
(n-3) / 2
\end{array}\right) 2^{-(n+3) / 2}(n+3) / 2-\left(\begin{array}{c}
n \\
(n+1) / 2
\end{array}\right) 2^{-(n+1) / 2}(n+1) / 2\right)\right)\right)\right) \text {. }
\end{aligned}
$$

We now proceed to derive the expected average sensitivity $\hat{s}_{2}^{f}$ of a typical function from $M_{0,2}^{1}(n, r, z, v)$, following the same steps.

Again, we only need to concern ourselves with four cases:

1. $\tilde{x} \in E^{n,(n-1) / 2}, f(\tilde{x})=1, x_{j}=1$

2. $\tilde{x} \in E^{n,(n-1) / 2}, f(\tilde{x})=0, x_{j}=0$

3. $\tilde{x} \in E^{n,(n+3) / 2}, f(\tilde{x})=1, x_{j}=1$

4. $\tilde{x} \in E^{n,(n+3) / 2}, f(\tilde{x})=0, x_{j}=0$

There are

$$
\frac{1}{2}\left(\begin{array}{c}
n \\
(n-1) / 2
\end{array}\right) 2^{-(n+1) / 2}
$$

minimal ones on $E^{n,(n-1) / 2}$ such that $x_{j}=1$. At the same time, there are

$$
\frac{1}{2}\left(\begin{array}{c}
n \\
(n-1) / 2
\end{array}\right)\left(1-2^{-(n+1) / 2}\right)
$$


zeros on $E^{n,(n-1) / 2}$ such that $x_{j}=0$. As for $M_{0,1}^{1}(n, r, z, v)$, the number of ones and zeros on $E^{n,(n+1) / 2}$ is not equal. The number of ones on $E^{n,(n+1) / 2}$ is given in (11). Thus, if $\tilde{x}^{(j, 0)}$ is a zero on $E^{n,(n-1) / 2}$, then the probability that $f\left(\tilde{x}^{(j, 1)}\right)=1$ is

$$
\begin{aligned}
\left(\begin{array}{c}
n \\
(n+1) / 2
\end{array}\right)^{-1}\left(\frac { 1 } { 2 } \left(\left(\begin{array}{c}
n \\
(n+1) / 2
\end{array}\right)+\left(\begin{array}{c}
n \\
(n-1) / 2
\end{array}\right)\right.\right. & 2^{-(n+1) / 2}(n-1) / 2 \\
& \left.\left.-\left(\begin{array}{c}
n \\
(n+3) / 2
\end{array}\right) 2^{-(n+3) / 2}(n+3) / 2\right)\right)
\end{aligned}
$$

where we have simply divided the number of ones on $E^{n,(n+1) / 2}$ by the total number of vectors on that layer. Thus, multiplying (30) by (31) and adding to (29) gives us the total contribution to the activity of variable $x_{j}$ from cases 1 and 2 above, which is equal to

$$
\begin{array}{r}
\frac{1}{2}\left(\begin{array}{c}
n \\
(n-1) / 2
\end{array}\right) 2^{-(n+1) / 2}+\frac{1}{2}\left(\begin{array}{c}
n \\
(n-1) / 2
\end{array}\right)\left(1-2^{-(n+1) / 2}\right) \times \\
\left(\begin{array}{c}
n \\
(n+1) / 2
\end{array}\right)^{-1}\left(\begin{array}{r}
\frac{1}{2}\left(\left(\begin{array}{c}
n \\
(n+1) / 2
\end{array}\right)+\left(\begin{array}{c}
n \\
(n-1) / 2
\end{array}\right) 2^{-(n+1) / 2}(n-1) / 2\right. \\
\left.\left.-\left(\begin{array}{c}
n \\
(n+3) / 2
\end{array}\right) 2^{-(n+3) / 2}(n+3) / 2\right)\right)
\end{array}\right.
\end{array}
$$

Cases 3 and 4 are similar. The number of (maximal) zeros on $E^{n,(n+3) / 2}$ for which $x_{j}=0$ is equal to

$$
\frac{1}{2}\left(\begin{array}{c}
n \\
(n+3) / 2
\end{array}\right) 2^{-(n+3) / 2}
$$

and the number of ones on $E^{n,(n+3) / 2}$ for which $x_{j}=1$ is equal to

$$
\frac{1}{2}\left(\begin{array}{c}
n \\
(n+3) / 2
\end{array}\right)\left(1-2^{-(n+3) / 2}\right)
$$

The proportion of zeros on $E^{n,(n+1) / 2}$ is

$$
\begin{aligned}
& \left(1-\left(\begin{array}{c}
n \\
(n+1) / 2
\end{array}\right)^{-1}\left(\frac { 1 } { 2 } \left(\left(\begin{array}{c}
n \\
(n+1) / 2
\end{array}\right)+\right.\right.\right. \\
& \left.\left.\left.\left(\begin{array}{c}
n \\
(n-1) / 2
\end{array}\right) 2^{-(n+1) / 2}(n-1) / 2-\left(\begin{array}{c}
n \\
(n+3) / 2
\end{array}\right) 2^{-(n+3) / 2}(n+3) / 2\right)\right)\right)
\end{aligned}
$$

Thus, as before, multiplying (34) by (35) and adding to (33), we get the total contribution to the activity from above and below the layer $E^{n,(n+3) / 2}$ (cases 3 and 4), resulting in

$$
\begin{aligned}
& \frac{1}{2}\left(\begin{array}{c}
n \\
(n+3) / 2
\end{array}\right) 2^{-(n+3) / 2}+ \frac{1}{2}\left(\begin{array}{c}
n \\
(n+3) / 2
\end{array}\right)\left(1-2^{-(n+3) / 2}\right) \times \\
&\left(1-\left(\begin{array}{c}
n \\
(n+1) / 2
\end{array}\right)\right.\left(\frac { 1 } { 2 } \left(\left(\begin{array}{c}
n \\
(n+1) / 2
\end{array}\right)+\right.\right. \\
&\left.\left.\left.\left(\begin{array}{c}
n \\
(n-1) / 2
\end{array}\right) 2^{-(n+1) / 2}(n-1) / 2-\left(\begin{array}{c}
n \\
(n+3) / 2
\end{array}\right) 2^{-(n+3) / 2}(n+3) / 2\right)\right)\right)
\end{aligned}
$$


Finally, adding (32) and (36) and then multiplying by $n 2^{-n+1}$ as in Theorem 4, we obtain the expected average sensitivity $\hat{s}_{2}^{f}$ of a typical function from $M_{0,2}^{1}(n, r, z, v)$ :

$$
\begin{aligned}
& \hat{s}_{2}^{f} \sim n 2^{-n+1}\left(\frac{1}{2}\left(\begin{array}{c}
n \\
(n-1) / 2
\end{array}\right) 2^{-(n+1) / 2}+\frac{1}{2}\left(\begin{array}{c}
n \\
(n-1) / 2
\end{array}\right)\left(1-2^{-(n+1) / 2}\right) \times\right. \\
& \left(\begin{array}{c}
n \\
(n+1) / 2
\end{array}\right)^{-1}\left(\frac { 1 } { 2 } \left(\left(\begin{array}{c}
n \\
(n+1) / 2
\end{array}\right)+\left(\begin{array}{c}
n \\
(n-1) / 2
\end{array}\right) 2^{-(n+1) / 2}(n-1) / 2\right.\right. \\
& \left.\left.-\left(\begin{array}{c}
n \\
(n+3) / 2
\end{array}\right) 2^{-(n+3) / 2}(n+3) / 2\right)\right)+ \\
& \frac{1}{2}\left(\begin{array}{c}
n \\
(n+3) / 2
\end{array}\right) 2^{-(n+3) / 2}+\frac{1}{2}\left(\begin{array}{c}
n \\
(n+3) / 2
\end{array}\right)\left(1-2^{-(n+3) / 2}\right) \times \\
& \left(1-\left(\begin{array}{c}
n \\
(n+1) / 2
\end{array}\right)^{-1}\left(\frac { 1 } { 2 } \left(\left(\begin{array}{c}
n \\
(n+1) / 2
\end{array}\right)+\right.\right.\right. \\
& \left.\left.\left.\left.\left(\begin{array}{c}
n \\
(n-1) / 2
\end{array}\right) 2^{-(n+1) / 2}(n-1) / 2-\left(\begin{array}{c}
n \\
(n+3) / 2
\end{array}\right) 2^{-(n+3) / 2}(n+3) / 2\right)\right)\right)\right) \text {. }
\end{aligned}
$$

Given that a function is equally likely to be picked from $M_{0,1}^{1}(n, r, z, v)$ as from $M_{0,2}^{1}(n, r, z, v)$, the expected average sensitivity is the average of Equations (28) and (37).

Funding: This research received no external funding.

Acknowledgments: I am grateful to the Institute for Systems Biology (ISB) for their support.

Conflicts of Interest: The author declares no conflict of interest.

\section{References}

1. Kauffman, S.A. Metabolic stability and epigenesis in randomly constructed genetic nets. J. Theor. Biol. 1969, 22, 437-467. [CrossRef]

2. Kauffman, S.A. The Origins of Order: Self-Organization and Selection in Evolution; Oxford University Press: Oxford, UK, 1993.

3. Derrida, B.; Pomeau, Y. Random networks of automata: A simple annealed approximation. EPL (Europhys. Lett.) 1986, 1, 45. [CrossRef]

4. Luque, B.; Solé, R.V. Lyapunov exponents in random Boolean networks. Physica A 2000, $284,33-45$. [CrossRef]

5. Shmulevich, I.; Kauffman, S.A. Activities and sensitivities in Boolean network models. Phys. Rev. Lett. 2004, 93, 048701. [CrossRef] [PubMed]

6. Munoz, M.A. Colloquium: Criticality and dynamical scaling in living systems. Rev. Mod. Phys. 2018, 90, 031001. [CrossRef]

7. Torres-Sosa, C.; Huang, S.; Aldana, M. Criticality Is an Emergent Property of Genetic Networks that Exhibit Evolvability. PLoS Comput. Boil. 2012, 8, e1002669. [CrossRef]

8. Krawitz, P.M.; Shmulevich, I. Basin Entropy in Boolean Network Ensembles. Phys. Rev. Lett. 2007, 98. [CrossRef]

9. Nykter, M.; Larjo, A.; Aho, T.; Kauffman, S.A.; Yli-Harja, O.; Shmulevich, I.; Price, N.D. Critical Networks Exhibit Maximal Information Diversity in Structure-Dynamics Relationships. Phys. Rev. Lett. 2008, 100. [CrossRef]

10. Ribeiro, A.S.; Kauffman, S.A.; Lloyd-Price, J.; Samuelsson, B.; Socolar, J.E.S. Mutual information in random Boolean models of regulatory networks. Phys. Rev. E 2008, 77. [CrossRef]

11. Galas, D.J.; Nykter, M.; Carter, G.W.; Price, N.D.; Shmulevich, I. Biological Information as Set-Based Complexity. IEEE Trans. Inf. Theory 2010, 56, 667-677. [CrossRef]

12. Kang, C.; Aguilar, B.; Shmulevich, I. Emergence of diversity in homogeneous coupled Boolean networks. Phys. Rev. E 2018, 97, 052415. [CrossRef] [PubMed] 
13. Goudarzi, A.; Teuscher, C.; Gulbahce, N.; Rohlf, T. Emergent Criticality through Adaptive Information Processing in Boolean Networks. Phys. Rev. Lett. 2012, 108. [CrossRef] [PubMed]

14. Echlin, M.; Aguilar, B.; Notarangelo, M.; Gibbs, D.L.; Shmulevich, I. Flexibility of Boolean Network Reservoir Computers in Approximating Arbitrary Recursive and Non-Recursive Binary Filters. Entropy 2018, $20,954$. [CrossRef]

15. Korshunov, A.D. Monotone boolean functions. Russ. Math. Surv. 2003, 58, 929-1001. [CrossRef]

16. Aracena, J.; Gadouleau, M.; Richard, A.; Salinas, L. Fixing monotone Boolean networks asynchronously. Inf. Comput. 2020, 104540. [CrossRef]

17. Aracena, J.; Richard, A.; Salinas, L. Number of fixed points and disjoint cycles in monotone Boolean networks. SIAM J. Discret. Math. 2017, 31, 1702-1725. [CrossRef]

18. Aracena, J.; Demongeot, J.; Goles, E. On limit cycles of monotone functions with symmetric connection graph. Theor. Comput. Sci. 2004, 322, 237-244. [CrossRef]

19. Melliti, T.; Regnault, D.; Richard, A.; Sené, S. Asynchronous simulation of Boolean networks by monotone Boolean networks. In International Conference on Cellular Automata; Springer: Cham, Switzerland, 2016; pp. 182-191.

20. Mesot, B.; Teuscher, C. Critical values in asynchronous random boolean networks. In European Conference on Artificial Life; Springer: Berlin/Heidelberg, Germany, 2003; pp. 367-376.

21. Shmulevich, I. Average sensitivity of typical monotone Boolean functions. arXiv 2005, arXiv:math/0507030.

22. Korshunov, A. On the number of monotone Boolean functions. Probl. Kibern. 1981, 38, 5-108.

23. Harris, S.E.; Sawhill, B.K.; Wuensche, A.; Kauffman, S. A model of transcriptional regulatory networks based on biases in the observed regulation rules. Complexity 2002, 7, 23-40. [CrossRef]

24. Serra, R.; Villani, M.; Semeria, A. Genetic network models and statistical properties of gene expression data in knock-out experiments. J. Theor. Biol. 2004, 227, 149-157. [CrossRef] [PubMed]

25. Li, Y.; Adeyeye, J.O.; Murrugarra, D.; Aguilar, B.; Laubenbacher, R. Boolean nested canalizing functions: A comprehensive analysis. Theor. Comput. Sci. 2013, 481, 24-36. [CrossRef]

26. Shmulevich, I.; Lähdesmäki, H.; Dougherty, E.R.; Astola, J.; Zhang, W. The role of certain Post classes in Boolean network models of genetic networks. Proc. Natl. Acad. Sci. USA 2003, 100, 10734-10739. [CrossRef] [PubMed]

27. Kahn, J.; Kalai, G.; Linial, N. The Influence of Variables on Boolean Functions. In Proceedings of the 29th Annual Symposium on Foundations of Computer Science, SFCS '88, White Plains, NY, USA, 24-26 October 1988; pp. 68-80, doi:10.1109/SFCS.1988.21923. [CrossRef]

28. Shmulevich, I.; Dougherty, E.R.; Kim, S.; Zhang, W. Probabilistic Boolean networks: A rule-based uncertainty model for gene regulatory networks. Bioinformatics 2002, 18, 261-274. [CrossRef] [PubMed]

29. Friedgut, E.; Kalai, G. Every monotone graph property has a sharp threshold. Proc. Am. Math. Soc. 1996, 124, 2993-3002. [CrossRef]

30. Bernasconi, A.; Damm, C.; Shparlinski, I. The average sensitivity of square-freeness. Comput. Complex. 2000, 9, 39-51. [CrossRef]

31. Bernasconi, A. Sensitivity vs. block sensitivity (an average-case study). Inf. Process. Lett. 1996, 59, $151-157$. [CrossRef]

32. Boppana, R.B. The average sensitivity of bounded-depth circuits. Inf. Process. Lett. 1997, 63, $257-261$. [CrossRef]

33. Friedgut, E. Boolean functions with low average sensitivity depend on few coordinates. Combinatorica 1998, 18, 27-35. [CrossRef]

34. Shi, Y. Lower bounds of quantum black-box complexity and degree of approximating polynomials by influence of Boolean variables. Inf. Process. Lett. 2000, 75, 79-83. [CrossRef]

35. Mossel, E.; O'Donnell, R. On the noise sensitivity of monotone functions. Random Struct. Algorithms 2003, 23, 333-350. [CrossRef]

36. Zhang, S. Note on the average sensitivity of monotone Boolean functions. Available online: http://www. cse.cuhk.edu.hk/ syzhang/papers/Sensitivity.pdf (accessed on 1 June 2020).

37. Bshouty, N.H.; Tamon, C. On the Fourier spectrum of monotone functions. J. ACM (JACM) 1996, 43, 747-770. [CrossRef]

38. Korshunov, A.D.; Shmulevich, I. On the distribution of the number of monotone Boolean functions relative to the number of lower units. Discret. Math. 2002, 257, 463-479. [CrossRef] 
39. Shmulevich, I.; Sellke, T.; Gabbouj, M.; Coyle, E. Stack filters and free distributive lattices. In Proceedings of the 1995 IEEE Workshop on Nonlinear Signal and Image Processing, Neos Marmaras, Greece, 20-22 June 1995; pp. 829-930.

40. Korshunov, A.D.; Shmulevich, I. The number of special monotone Boolean functions and statistical properties of stack filters. Diskretn. Anal. Issled. Oper. 2000, 7, 17-44.

41. Shmulevich, I.; Yli-Harja, O.; Astola, J.; Korshunov, A. On the robustness of the class of stack filters. IEEE Trans. Signal Process. 2002, 50, 1640-1649. [CrossRef]

(C) 2020 by the author. Licensee MDPI, Basel, Switzerland. This article is an open access article distributed under the terms and conditions of the Creative Commons Attribution (CC BY) license (http:// creativecommons.org/licenses/by/4.0/). 